\title{
The resistant macular oedema
}

\section{Malla OK}

Professor and Head of Department, Department of Ophthalmology, Kathmandu Medical College, Sinamangal, Nepal.

$\mathrm{T}^{\mathrm{s}}$ he retina is the membranous light sensitive innermost coat of the eye. The central part of the retina is called macula and it is responsible for the acuity of vision.

Macular Oedema (MO) is a common problem of retina and an important cause of visual disturbance. In this condition there is an increased accumulation of fluid within the intraretinal layers of the macula as a result of microvascular changes and breakdown of blood-retinal barrier.

Visual loss in Diabetic Retinopathy (DR) and Retinal Vein Occlusion (RVO), the two commonest retinal conditions, is mostly due to MO. Other less common causes are uveitis, postsurgery and retinitis pigmentosa.

Prostaglandins may be the cause of MO in association with anterior segment surgery or inflammation. Posterior diffusion of one or more of the eosamoids/metabolites through the vitreous is assumed to alter capillary permeability of the perifoveal network leading to the characteristic pattern of intraretinal fluid accumulation and cyst formation. MO may occur in $50 \%$ of patients with intermediate uveitis and is a hallmark of pars planitis. Optical Coherance Tomography (OCT) in MO include diffuse retinal thickening with cystic areas of low reflectivity which is more pronounced in the outer plexiform layers.

The incidence of MO following cataract surgery has greatly reduced. With the advent of newer techniques of phacosurgery where the posterior capsule remains intact, most cases of MO are mild and asymptomatic. There has also been noted a spontaneous resolution clinically in over $90 \%$ of uncomplicated cases usually within six months. More severe MO may result in permanent visual loss, incidence increasing with significant postoperative uveitis with surgical/ IntraOcular Lens (IOL) complications such as vitreous loss or iris prolapse.

Inflammation is important in the causation of $\mathrm{MO}$, as evidenced by association with varying degrees of uveitis/vitritis and retinal phlebitis. Other important clinical associations include systemic vascular disease such as hypertension and diabetes mellitus. The above mentioned conditions resulting in MO have largely been addressed in Nepal too except for in DR and RVO.

DR and RVO are common retinal vascular diseases seen in Retina Clinics in Nepal. In these conditions MO is the end result in many cases with profound loss of vision.

Management of MO in DR and RVO has lately been a challenge to retinal surgeons. The standard of care for MO is laser photocoagulation. A substantial group of patients, however, are unresponsive to laser therapy and fail to improve after photocoagulation ${ }^{1}$. Newer methods and modalities have been evaluated for treating MO in DR and RVO including the use of combination treatments involving laser PC, intraocular injection of steroids and therapeutics targeting vascular endothelial growth factor (VEGF). Monotherapy for the underlying $\mathrm{MO}$ is also under investigation with steroids and antiVEGF agents.

Favourable results have been reported with intravitreal administration of Triamcinolone acetonamide (TA) in the treatment of MO persistent despite laser photocoagulation. However, intravitreal TA shows a limited duration of action with the need of multiple injections and carries the implicit risks of repeated procedures and serious complications, including vitreous haemorrhage, retinal detachment and endophthalmitis ${ }^{2}$.

Recently these modes of treatment e.g. intravitreal injections of steroids (Triamcinolone acetonamide) and VEGF agents (Avastin) have been tried in different centres in Nepal. However, there are no long term studies on the effects of intravitreal Avastin. At the same time in a country like Nepal where the GNI per capita is US \$ 340 (2007) and 5.5 percent of the population fall below international poverty line of US $\$ 1.25$ per day $(2005)^{3}$, Avastin is not affordable by the common people. Very recently FDA (Food and Drug Administration, USA) has approved Ozurdex, the first drug therapy for the treatment of macular oedema following RVO. 
All said managing a case of MO in DR and RVO is still a challenge. Many research works are going on but till date none have been conclusive.

\section{References}

1. Lee CM, Olk RJ. Modified grid laserphotocoagulation for diffuse diabetic macular oedema.Long term results. Ophthalmology.1991;98:1594-602.
2. Wu L. Martinez-Castellanos MA, QuirozMercado $\mathrm{H}$, et al. Twelve-month safety of Intravitreal injections of bevacizumab (Avastin): results of the Pan-American Collaborative Retina Study Group (PACORES). Graefes Arch Clin Exp Ophthalmol. 2008;246(1): 81-7.

3. Unicef. Unite for chidren [homepage on the internet]. Geneva: UNICEF; [updated on 2005]. Available from: www.unicef.org/infobycountry/ nepal_nepal.statistics.htm 